\title{
ON THE DISTRIBUTION OF THE VALUES OF THE PARTIAL SUMS OF A TAYLOR SERIES
}

\author{
V. F. COWLING
}

1. Introduction. The object of this paper is to determine restrictions on the coefficients $a_{n}$ of a Taylor series which, when taken together with the fact that the complex variable $z$ lies in certain regions of the complex plane, will imply that the values of the partial sums $S_{n}(z)=a_{0}+a_{1} z+\cdots+a_{n} z^{n}, n=0,1,2, \cdots$, of a Taylor series will be numbers lying in a predetermined region $V$ of the complex plane. If the region $V$ does not contain the origin, we then have some information about the zero free regions of polynomials and other classes of functions. The method that is employed is an adaptation of a technique developed principally by Leighton and Thron [3] ${ }^{1}$ in the study of convergence criteria for continued fractions.

2. Two lemmas. We employ the same notation and terminology here as in [1]. If $S$ is a set of complex numbers and $a$ is a fixed complex number, we shall designate by $a+S$ and $S+a$ the set obtained from the set $S$ by adding to each element of $S$ the number $a$. By $a S$ we understand the set obtained from $S$ by multiplying each element of $S$ by the number $a$. By the notation $D[a S]$ is understood the point set intersection of all sets $a S$ as $a$ assumes all values in a given set $A$.

LEMMA 2. 1. Let $E, V$, and $Z$ be any three sets of complex numbers with the following properties:

(a) $1+E \subset V$;

(b) $1+a v \in V$, if $a \in E$ and $v \in V$.

Let $a_{n} \neq 0, n=0,1,2, \cdots$. If the quantities $a_{n} a_{n-1}^{-1} z \in E$ for all $z \in Z$ and each $n=1,2, \cdots$, the values of the partial sums $S_{n}(z)=a_{0}+a_{1} z$ $+\cdots+a_{n} z^{n}, n=1,2, \cdots$, of a Taylor series are numbers which are contained in $a_{0} V$.

Proof. We note that

$$
\begin{aligned}
S_{n}(z) & =a_{0}+a_{1} z+\cdots+a_{n} z^{n} \\
& =a_{0}\left\{1+\frac{a_{1}}{a_{0}} z\left\{1+\frac{a_{2}}{a_{1}} z\left\{\cdots \frac{a_{n-1}}{a_{n-2}} z\left\{1+\frac{a_{n}}{a_{n-1}} z\right\} \cdots\right\} .\right.\right.
\end{aligned}
$$

Now $1+a_{n} a_{n-1}^{-1} z \in V$ for $z \in Z$ and $n=1,2, \cdots$ by (a) and hence $1+a_{n-1} a_{n-2}^{-1} z\left\{1+a_{n} a_{n-1}^{-1} z\right\} \in V$ by (b). A simple induction argument

Received by the editors October 26, 1950.

${ }^{1}$ Numbers in brackets refer to the bibliography at the end of the paper. 
then proves that $S_{n}(z) \in a_{0} V, n=1,2, \cdots$, for $z \in Z$.

Leмma 2.2. Let $V$ and $Z$ be any sets of complex numbers such that the set $E=D\left[v^{-1}(V-1)\right], v \in V$, is not empty, and has the property that $1+E \subset V$. If the quantities $a_{n} a_{n-1}^{-1} z \in E$ for $z \in Z$ and each $n$ $=1,2, \cdots$, the values of the partial sums $S_{n}(z)=a_{0}+a_{1} z+\cdots$ $+a_{n} z^{n}, n=1,2, \cdots$, of a Taylor series are numbers which are contained in $a_{0} V$.

Proof. To prove the lemma it is sufficient to show that the condition (b) of Lemma 2.1 is satisfied. Let $a$ be any point belonging to $E$ and $v$ any point of $V$; then the definition of $E$ insures the existence of a point $v^{\prime} \in V$ such that $a=v^{-1}\left(v^{\prime}-1\right)$, that is, $v^{\prime}=1+a v \in V$ and condition (b) of Lemma 2.1 is satisfied. As an immediate consequence of Lemma 2.2 we have the following corollary.

CoRollary 2.1. Let $V$ and $Z$ be any two sets of complex numbers such that the set $E=D\left[v^{-1}(V-1)\right], v \in V$, is not empty and 1 is contained in $V$. Then the conclusion of Lemma 2.2 holds including the case $n=0$.

3. Applications. We shall now prove three theorems which illustrate the use of this method. From the nature of the technique to be used it seems that the greatest difficulty one encounters is in the actual problem of trying to obtain an expression for the boundary of $E$.

Let us choose first for the set $V$ of Lemma 2.2 the region $|z-(1-p) / 2| \geqq(1+p) / 2,0<p \leqq 1$. Then $V-1$ becomes the region $|z+(1+p) / 2| \geqq(1+p) / 2$. If $v \in V$, then $v^{-1}$ is contained in the circular region $|z-(p-1) / 2 p| \leqq(p+1) / 2 p$ and we are interested in determining $E=D\left[v^{-1}(V-1)\right]$. However this is really the problem considered in $[1$, p. 354] but with different constants involved. Thus a straightforward application of the method involved in [1] reveals that $E=D\left[v^{-1}(V-1)\right]$ is the set of points $z=r e^{i \theta}$ for which

(3.1) $\quad r \geqq\left[\left(p^{2}-1\right) / 2 p\right] \cos \theta+(p+1)^{2} / 2 p, \quad 0<p \leqq 1$ (fixed).

It may be of interest to note that $E$ is the set of points common to a family of circles having their centers on the circle $\left|z-\left(p^{2}-1\right) 4 p\right|$ $\leqq(p+1)^{2} / 4 p$ and passing through the origin. The envelope of this family of circles is the curve determined by (3.1). Although this does not prove that (3.1) is the boundary of $E$, it provides a motivation for considering this expression and then showing by direct methods as in [1] that it actually is the boundary of $E$. This completes the proof of the following theorem.

THEOREM 3.1. If for some $0<p \leqq 1$ the quantities $a_{n} a_{n-1}^{-1} z$ are con- 
tained in the region $r \geqq\left[\left(p^{2}-1\right) / 2 p\right] \cos \theta+(p+1)^{2} / 2 p, 0<\theta \leqq 2 \pi$, for all $z$ in some set $Z$ and each $n=1,2, \cdots$, then the values of the partial sums $S_{n}(z)=a_{0}+a_{1} z+\cdots+a_{n} z^{n}, n=0,1,2, \cdots$, are contained in the region $\left|S_{n}(z)-(1-p) / 2\right| \geqq(1+p) / 2$ multiplied by $a_{0}$.

The following corollaries are immediate consequences of the theorem just proved.

COROLLARY 3.1. Let $z=r e^{i \theta}$. If $1 \leqq a_{n} a_{n-1}^{-1}$ for each $n=1,2, \cdots$, and $r \geqq\left[\left(p^{2}-1\right) / 2 p\right] \cos \theta+(p+1)^{2} / 2 p, 0<\theta \leqq 2 \pi$, for fixed $0<p \leqq 1$, then the conclusion of Theorem 3.1 holds.

If now we set $p=1$ in Theorem 3.1 we have the following corollary.

COROLI.ARY 3.11. The polynomial $a_{0}+a_{1} z+\cdots+a_{n} z^{n}, a_{0} \neq 0$, has all its roots interior to the circle $|z| \leqq 2 \max \left[\left|a_{0} / a_{1}\right|, \cdots,\left|a_{n-1} / a_{n}\right|\right]$. Actually $\left|S_{k}(z)\right| \geqq\left|a_{0}\right|$, for $k=0,1,2, \cdots, n$, in $|z| \geqq 2 \max \left[\left|a_{0} / a_{1}\right|\right.$, ..., $\left.\left|a_{n-1} / a_{n}\right|\right]$.

This last result, however, is not quite so strong as that found in [2, p. 18]. Of course Theorem 3.1 itself can be interpreted as a result about the zeros of polynomials.

We choose next for the set $V$ of Lemma 2.2 the region common to the regions $\operatorname{Re}(z) \geqq p, 0<p \leqq 1$, and $|z-1| \leqq R, R>1$. Then $V-1$ becomes the region common to $\operatorname{Re}(z) \geqq p-1,0<p \leqq 1$, and $|z| \leqq R$. If $v \in V$, then $v^{-1}$ is contained in the region $V^{-1}$ common to $|z-1 / 2 p|$ $\leqq 1 / p$ and $\left|z-1 /\left(1-R^{2}\right)\right| \geqq R /\left(R^{2}-1\right)$. Now we wish to determine $E=E(p, R)=D\left(v^{-1}(V-1)\right], v \in V$. To this end we show first that $D\left[v^{-1}(V-1)\right]=D\left[v_{1}^{-1}(V-1)\right]$ where $v_{1}^{-1}$ lies on the arc $C$ of a circle $\left|z+1 /\left(R^{2}-1\right)\right|=R /\left(R^{2}-1\right),|\operatorname{Arg} z| \leqq \operatorname{Arc} \operatorname{Cos} p /(R+2 p-1)^{1 / 2}$. If we choose a $v^{-1} \in V^{-1}$, a $v_{1}^{-1}$ on the arc $C$ can be found with the property that $\left|v_{1}^{-1}\right| \leqq\left|v^{-1}\right|$ and $\operatorname{Arg} v_{1}^{-1}=\operatorname{Arg} v^{-1}$. Thus since $0 \in V-1$, the set $v_{1}^{-1}(V-1)$ is contained in the set $v^{-1}(V-1)$ and hence $D\left[v_{1}^{-1}(V-1)\right]=D\left[v^{-1}(V-1)\right]$.

Now we note that $E(p, R)$ may be obtained by considering it as the set common to the result obtained in case (a) $V-1$ is the region $\operatorname{Re}(z) \geqq p-1$ and (b) $V-1$ is the region $|z| \leqq R$.

Let us consider case (b) first. In this case a simple inspection shows that $E_{b}=E_{b}(R)$ is the set obtained by multiplying the region $|z| \leqq R$ by the complex number of minimum modulus on the arc $C$. Thus $E_{b}$ is the region $|z| \leqq R /(R+1)$. Now in case (a) a simple calculation reveals that $E_{a}=E_{a}(p, R)$ is the intersection of a family of right half-planes each of which is tangent to the arc $C^{\prime}$ of a circle

$$
\left|z+(1-p) /\left(R^{2}-1\right)\right| \leqq R(1-p) /\left(R^{2}-1\right)
$$




$$
|\operatorname{Arg} z-\pi| \leqq \operatorname{Arc} \operatorname{Cos} p /(R+2 p-1)^{1 / 2} .
$$

Thus $E_{a}=E_{a}(p, R)=D\left[v_{1}^{-1}(V-1)\right]$ in this case consists of the arc $C^{\prime}$ plus the two right half-lines extending to infinity which are tangent to the circle $C^{\prime}$ at the end points of the arc indicated above. The intersection of the regions obtained in cases (a) and (b) is then our region $E=E(p, R)$, that is, $E(p, R)=E_{b}(R) \cap E_{a}(p, R)$. We then have the following theorem.

THEOREM 3.2. If for some $0<p \leqq 1$ and some $R>1$ the quantities $a_{n} a_{n-1}^{-1} z \in E(p, R)$ for all $z$ contained in some set $Z$ and each $n=1,2, \cdots$, then the values of the partial sums $S_{n}(z)=a_{0}+a_{1} z+\cdots+a_{n} z^{n}$, $n=0,1, \cdots$, lie in the region common to $\operatorname{Re}(z) \geqq p$ and $|z-1| \leqq R$ multiplied by $a_{0}$.

If now $0<a_{n} a_{n-1}^{-1} \leqq 1$ (in this case the radius of convergence of the corresponding Taylor series is not less than 1) we have the following corollary.

CoROLlaRY 3.2. If $0<a_{n} a_{n-1}^{-1} \leqq 1, n=1,2, \cdots$, and $z \in E(p, R)$, the conclusion of Theorem 3.2 holds.

This last corollary may of course be given the following interpretation: namely, if $0<a_{k} a_{k-1}^{-1} \leqq 1$ for $k=1,2, \cdots, n$, then the polynomial $a_{0}+a_{1} z+\cdots+a_{n} z^{n}$ does not vanish for $z \in E(p, R)$ for any $0<p \leqq 1$ and any $R>1$.

Finally we choose for the set $V$ of Lemma 2.2 the region $|z-k / 2|$ $\leqq k / 2, k>1$. $V-1$ is then the circular region $|z-(k / 2-1)| \leqq k / 2$. We show first that the region $E(k)=D\left[v^{-1}(V-1)\right]$, where $v_{1}^{-1}$ ranges over $\operatorname{Re}(z) \geqq 1 / k$, is equal to the region $D\left[v_{1}^{-1}(V-1)\right]$, where $v_{1}^{-1}$ ranges over $\operatorname{Re}(z)=1 / k$. In order to do this it is sufficient to show that for any set $v^{-1}(V-1)$ a set $v_{1}^{-1}(V-1)$ contained in $v^{-1}(V-1)$ can be found where $v_{1}^{-1}$ is a point on $\operatorname{Re}(z)=1 / k$. We can find such a number by taking the number lying on $\operatorname{Re}(z)=1 / k$ and satisfying the condition $\operatorname{Arg} v_{1}^{-1}=\operatorname{Arg} v^{-1}$.

The set $V-1$ is subjected to the same rotation by multiplication by $v^{-1}$ as by $v_{1}^{-1}$ and, since $0 \in v^{-1}(V-1)$, the stretching effected by $\left|v^{-1}\right| \geqq\left|v_{1}^{-1}\right|$ insures that the set $v^{-1}(V-1) \supset v_{1}^{-1}(V-1)$.

Consider the circular region $|z-(k / 2-1)| \leqq k / 2$. If we now make the linear transformation $w=a z$ where $\operatorname{Re}(a)=1 / k$, this circular region becomes the new circular region $|z-a(k / 2-1)| \leqq(k / 2)|a|$ or, noting that $a=(1 / k) \sec \theta e^{i \theta}$, this becomes

$$
\left|z-\frac{\sec \theta}{k} e^{i \theta}\left(\frac{k}{2}-1\right)\right|=\frac{\sec \theta}{2},-\frac{\pi}{2} \leqq \theta \leqq \frac{\pi}{2} \text {. }
$$


The set $E(k)=D\left[v^{-1}(V-1)\right]$ is then the common set to all the circles (3.2) as $\theta$ varies over the range $-\pi / 2 \leqq \theta \leqq \pi$. We now proceed to determine the envelope of the family of circles (3.2) and then show directly that this envelope is the boundary of the region $E(k)$. For fixed $k>1,(3.2)$ then represents a family of circles with centers on the line $\operatorname{Re}(z)=(1 / 2)-(1 / k)$, each circle passing through a point on the line $\operatorname{Re}(z)=1-1 / k$ having the same argument as the center of the circle.

Upon setting $z=x+i y$ in (3.2) we see that we are looking for the envelope of the family of circles ( $k$ fixed and greater than 1)

$$
\begin{aligned}
\left(x-\left(\frac{1}{2}-\frac{1}{k}\right)\right)^{2}+\left(y-\left(\frac{1}{2}-\frac{1}{k}\right)^{2} \tan \theta\right) & =\frac{\sec ^{2} \theta}{4}, \\
& -\pi / 2 \leqq \theta \leqq \pi / 2 .
\end{aligned}
$$

Differentiation with respect to $\theta$ leads to

$$
\tan \theta=\frac{k(k-2)}{2(1-k)} y .
$$

Upon eliminating $\theta$ between the equations (3.3) and (3.4) we obtain for the equation of the envelope ( $k>1$ and fixed)

$$
\left(x-\frac{k-2}{2 k}\right)^{2}+\frac{k^{2}}{4(k-1)} y^{2}=\frac{1}{4} .
$$

For each $k>1,(3.5)$ is the equation of an ellipse with major axis of unit length having the ends of its minor axis on the circle $x^{2}+y^{2}=1 / 2$.

Now in order to prove that (3.5) is the equation of the boundary of $E(k)(k>1$ and fixed) we shall show that if $p(x, y)$ is a point contained in the ellipse, then it will be contained in all the circles of the family (3.3). This will be sufficient, for clearly if $p(x, y)$ is outside the ellipse (3.5), it is outside at least one of the circles of the family (3.3). Thus we wish to show that

$$
\left(x-\frac{k-2}{2 k}\right)^{2}+\frac{k^{2}}{4(k-1)} y^{2} \leqq \frac{1}{4} \quad(k>1)
$$

implies

(3.7) $\left(x-\left(\frac{1}{2}-\frac{1}{k}\right)\right)^{2}+\left(y-\left(\frac{1}{2}-\frac{1}{k}\right) \tan \theta\right)^{2} \leqq \frac{\sec ^{2} \theta}{4}$,

$$
0<\theta \leqq 2 \pi \text {. }
$$

If now we add $(y-(1 / 2-1 / k) \tan \theta)^{2}$ to both members of (3.6), 


$$
\begin{aligned}
\left(x-\frac{k-2}{2 k}\right)^{2}+ & \left(y-\left(\frac{1}{2}-\frac{1}{k}\right) \tan \theta\right)^{2} \\
& \leqq \frac{1}{4}+\left(y-\left(\frac{1}{2}-\frac{1}{k}\right) \tan \theta\right)^{2}-\frac{k^{2}}{4(k-1)} y^{2}
\end{aligned}
$$

Thus in order to show that (3.6) implies (3.7) it will clearly be sufficient to show that

$$
-\frac{(k-2)^{2}}{4(k-1)}-2\left(\frac{1}{2}-\frac{1}{k}\right) \tan \theta y+\left(\frac{1}{k^{2}}-\frac{1}{k}\right) \tan ^{2} \theta \leqq 0
$$

for each $k>1$ and each $0<\theta \leqq 2 \pi$. But the left member of (3.9) may be written as $-(k-2)^{2} / 4(k-1)(y+(2(k-1) / k(k-2)) \tan \theta)^{2} \leqq 0$ for each $0<\theta \leqq 2 \pi$ and each $k>1$. This completes the proof that the boundary of $E(k)$ is given by (3.5) for each $k>1$. Thus we have the following theorem.

THEOREM 3.3. Let $S_{n}(z)=a_{0}+a_{1} z+\cdots+a_{n} z^{n}, n=0,1,2, \cdots$, where $z=x+i y$. If for some $k>1, a_{n} a_{n-1}^{-1} z \in E(k)$ for all $z$ contained in some set $Z$ and each $n=0,1,2, \cdots$, where $E(k)$ is the set of points interior to or on the boundary of the ellipse

$$
[x-(k-2) / 2 k]^{2}+\left[k^{2} / 4(k-1)\right] y^{2}=1 / 4,
$$

then $\left|S_{n}(z)-a_{0} k / 2\right| \leqq\left|a_{0}\right| k / 2$, for all $z \in Z$ and each $n=0,1,2, \cdots$.

The following corollary is an immediate consequence of the above theorem.

Corollary 3.3. If $z \in E(k)$ for some $k>1$ and if $0<a_{n} a_{n-1}^{-1} \leqq 1$, for each $n=0,1,2, \cdots$, then the values of the partial sums $S_{n}(z)=a_{0}$ $+a_{1} z+\cdots+a_{n} z^{n}, n=0,1,2, \cdots$, are contained in the half-plane $\operatorname{Re}\left(S_{n}(z)\right) \geqq 0$.

4. Conclusions. It seems clear that a large number of results of the nature of the above theorems could be obtained; however, we feel that the ones presented are sufficiently representative of the general situation. While the above lemmas and theorems are concerned only with Taylor series, it seems probable that similar results could be obtained for the general infinite series $u_{0}(z)+u_{1}(x)+\cdots+u_{n}(z)$ $+\cdots$. This remark is based on the observation that we may write

$$
\begin{aligned}
S_{n}(z) & =u_{0}(z)+u_{1}(z)+\cdots+u_{n}(z) \\
& =u_{0}(z)\left\{1+\frac{u_{1}(z)}{u_{0}(z)}\left\{1+\frac{u_{2}(z)}{u_{1}(z)}\left\{\cdots \frac{u_{n-1}(z)}{u_{n-2}(z)}\left\{1+\frac{u_{n}(z)}{u_{n-1}(z)} \cdots\right\} .\right.\right.\right.
\end{aligned}
$$




\section{BIBLIOGRAPHY}

1. V. F. Cowling, W. Leighton, and W. J. Thron, Twin convergence regions for continued fractions, Bull. Amer. Math. Soc. vol. 50 (1944) pp. 351-357.

2. J. Dieudonne, La theorie analytique des polynomes d'une variable, Memorial des Sciences Mathématiques, vol. 93, Paris, 1938.

3. W. Leighton and W. J. Thron, Continued fractions with complex elements, Duke Math. J. vol. 9 (1942) pp. 763-772.

The University of Kentucky

\section{NORMS OF MATRIX TYPE FOR THE SPACES OF CONVERGENT AND BOUNDED SEQUENCES}

\section{ALBERT WILANSKY}

Let, as usual, $x=\left\{x_{n}\right\} \in(c)$ mean that $x$ is a convergent sequence. We write $\|x\|=\|x\|_{A}=\sup _{n}\left|\sum_{k=1}^{n} a_{n k} x_{k}\right|=\sup _{n}\left|A_{n}(x)\right|$, where $A$ $=\left(a_{n k}\right)$ is a matrix of complex numbers. By the ordinary norm of $x$ we shall understand $|x|=\sup _{n}\left|x_{n}\right|$.

Problem 1. What conditions on $A$ are necessary and sufficient that (c) be a Banach space with this norm?

The first result is that $\|x\|<\infty$ for all $x \in(c)$ if and only if $\|A\|$ $=\sup _{n} \sum_{k=1}^{\infty}\left|a_{n k}\right|<\infty$ (essentially due to Toeplitz, 1911), and this is true if and only if $\|x\|<\infty$ for all $x \in(m)$, the space of bounded sequences.

We shall assume that $A$ is normal, that is, that $a_{n k}=0$ for $k>n$, while $a_{n n} \neq 0$. (A remark on reversibility will be appended.) Finally, let $(A)$ be the class of sequences $x$ such that $A x=\left\{A_{n}(x)\right\}$ is convergent.

Theorem 1. Let $A$ be normal, $\|A\|<\infty$, then for $(c)$ to be a Banach space it is sufficient that $\left\|A^{-1}\right\|<\infty$. The metric will then be equivalent to the ordinary metric. The condition is not necessary (even if $A$ is conservative).

(A conservative matrix-one with $(A) \supset(c)$-must satisfy $\|A\|$ $<\infty$, but not conversely.)

Assume that $A$ satisfies the hypotheses. For $x \in(A), y=A x$, we deduce that $\left|x_{n}\right| \leqq\left\|A^{-1}\right\| \cdot \sup _{n}\left|y_{n}\right|=\left\|A^{-1}\right\| \cdot\|x\|$, and conclude: (1) $(A) \subset(m) ;(2) L_{n}(x)=x_{n}$ defines an additive continuous functional $L_{n}$ on $(A)$.

Presented to the Society, February 24, 1951 under the title On norms of matrix type for $(c)$ and $(m)$; received by the editors October 16, 1950. 